EPHOU-99-002

February, 1999

\title{
Lattice Chern-Simons Gravity via Ponzano-Regge Model
}

\author{
Noboru Kawamoto ${ }^{1)}$ 丹, Holger Bech Nielsen ${ }^{2)}$ 自 and Noriaki Sato ${ }^{1)}$ f \\ 1) Department of Physics, Hokkaido University \\ Sapporo, 060-0810, Japan \\ 2) Niels Bohr Institute \\ Blegdamsvj 17, Copenhagen, Denmark
}

\begin{abstract}
We propose a lattice version of Chern-Simons gravity and show that the partition function coincides with Ponzano-Regge model and the action leads to the Chern-Simons gravity in the continuum limit. The action is explicitly constructed by lattice dreibein and spin connection and is shown to be invariant under lattice local Lorentz transformation and gauge diffeomorphism. The action includes the constraint which can be interpreted as a gauge fixing condition of the lattice gauge diffeomorphism.
\end{abstract}

\footnotetext{
*kawamoto@particle.sci.hokudai.ac.jp

${ }^{\dagger}$ HBECH@nbivms.nbi.dk

${ }_{\ddagger}^{\ddagger}$ noriaki@particle.sci.hokudai.ac.jp
} 


\section{Introduction}

It is not obvious that the string is the only formulation leading to the quantum gravity. In fact, two dimensional quantum gravity was formulated by a lattice gravity, the dynamical triangulation of random surface. On the other hand, three dimensional Einstein gravity was successfully formulated by the Chern-Simons action even at the quantum level[1]. It is natural but nontrivial expectation that the three dimensional gravity will be formulated on the lattice. In particular it is natural to ask how one can formulate the Chern-Simons gravity on the lattice.

Ponzano and Regge (P-R) proposed a 3-dimensional lattice gravity model based on $6-j$ symbols[2] about 30 years ago. At the early '90s Turaev and Viro (T-V) proposed $q$-deformed version of the P-R model[3] and then Turaev pointed out that the partition function of the $\mathrm{T}-\mathrm{V}$ model is the square of the partition function of $S U(2)$ Chern-Simons gauge theory which is equivalent to the Palatini action with a cosmological term[4]. [5]. On the other hand Ooguri and Sasakura showed that the P-R model is equivalent to the $I S O(3)$ Chern-Simons theory [5]. They showed the equivalence by using the wave function of Wheeler-DeWitt equation and the knowledge of conformal field theory. The proof is, however, indirect.

There is also another indirect approach to show the equivalence of the P-R model and Chern-Simons gravity. Vanishing curvature condition is the equation of motion of the Chern-Simons gravity and can be used to derive $6-j$ symbol via orthogonality of character [6] [7] [8]. Thus the equivalence is on the classical level.

Apart from these development two of the present authors (Kawamoto and Nielsen) proposed a gravity version of Wilson's lattice gauge theory 9 where the plaquette action plays a fundamental role. There was an independent proposal[10] similar to ours.

In this paper we extend the formulation previously proposed by the authors and

explicitly construct a lattice Chern-Simons gravity by identifying the location of dreibein and spin connection on a simplicial lattice manifold. After the integration of the dreibein and spin connection, we obtain the Ponzano-Regge model. We clarify the lattice version of local Lorentz transformation and the gauge diffeomorphism. We then give arguments that the lattice action leads to the Chern-Simons action in 
the continuum limit.

The standard Chern-Simons action is formulated by one form gauge field and zero form gauge parameter. Since the three dimensional Chern-Simons gravity is formulated only by forms, the general coordinate diffeomorphism invariance of the action is trivial and should be reflected on the lattice. The standard Chern-Simons action has been generalized into arbitrary dimensions [11 by introducing all the possible form degrees. It has been analysed that the two and four dimensional generalized Chern-Simons actions lead to a two dimensional topological gravity and four dimensional topological conformal gravity,respectively, at the classical level 12. One of the important aim of the current analysis of the lattice Chern-Simons gravity is to extend the formulation given here into other dimensions including four by using the generalized Chern-Simons actions.

\section{Brief Summary of Chern-Simons Gravity and Ponzano-Regge Model}

\subsection{Chern-Simons Gravity}

We first summarize the Chern-Simons gravity formulated by Witten[1]. We choose the gauge group as Euclidean version of three dimensional Poincare group ISO(3). Then we define one form gauge field and zero form gauge parameter as

$$
\begin{gathered}
A_{\mu}=e_{\mu}^{a} P_{a}+\omega_{\mu}^{a} J_{a}, \\
v=\rho^{a} P_{a}+\tau^{a} J_{a},
\end{gathered}
$$

where $e_{\mu}^{a}$ and $\omega_{\mu}^{a}$ are dreibein and spin connection, respectively, and $\rho$ and $\tau$ are the corresponding gauge parameters. The momentum generator $P_{a}$ and the angular momentum generator $J_{a}$ of $I S O(3)$ satisfy

$$
\left[J_{a}, J_{b}\right]=\epsilon_{a b c} J^{c}, \quad\left[J_{a}, P_{b}\right]=\epsilon_{a b c} P^{c}, \quad\left[P_{a}, P_{b}\right]=0
$$

Using the invariant quadratic form which is particular in three dimensions, we can define the inner product

$$
\left\langle J_{a}, P_{b}\right\rangle=\delta_{a b}, \quad\left\langle J_{a}, J_{b}\right\rangle=\left\langle P_{a}, P_{b}\right\rangle=0 .
$$


We then obtain Einstein-Hilbert action of three dimensional gravity from ChernSimons action

$$
\int\left\langle A d A+\frac{2}{3} A^{3}\right\rangle=\int \epsilon^{\mu \nu \rho} e_{\mu a} F_{\nu \rho}^{a} d^{3} x
$$

where

$$
F_{\mu \nu}^{a}=\partial_{\mu} \omega_{\nu}^{a}-\partial_{\nu} \omega_{\mu}^{a}+\epsilon_{b c}^{a} \omega_{\mu}^{b} \omega_{\nu}^{c}
$$

The component wise gauge transformation of $\delta A_{\mu}=-D_{\mu} v$ is given by

$$
\begin{aligned}
\delta e_{\mu}^{a} & =-D_{\mu} \rho^{a}-\epsilon^{a b c} e_{\mu b} \tau_{c} \\
\delta \omega_{\mu}^{a} & =-D_{\mu} \tau^{a} .
\end{aligned}
$$

At this stage it is important to recognize that the local Lorentz transformation is generated by the gauge parameter $\tau$

$$
\begin{aligned}
& \delta e_{\mu}^{a}=-\epsilon^{a b c} e_{\mu b} \tau_{c} \\
& \delta \omega_{\mu}^{a}=-D_{\mu} \tau^{a}
\end{aligned}
$$

while the gauge transformation of diffeomorphism is generated by the gauge parameter $\rho$

$$
\begin{aligned}
\delta e_{\mu}^{a} & =-D_{\mu} \rho^{a}, \\
\delta \omega_{\mu}^{a} & =0 .
\end{aligned}
$$

Three dimensional Einstein gravity is thus elegantly formulated by Chern-Simons action. This is essentially related to the fact that the three dimensional Einstein gravity does not include dynamical graviton and thus can be formulated by the topological Chern-Simons action. The equivalence of the above action and EinsteinHilbert action is, however, valid only if the dreibein $e_{\mu}^{a}$ is invertible. The quantization and perturbative renormalizability around the nonphysical classical background $e_{\mu}^{a}=0$ is the natural consequence of the formulation.

It has been pointed out that $I S O(3)$ Chern-Simons gravity action is equivalent to the Palatini action for three dimensional gravity, which is essentially the three dimensional version of $B F$ theory[13]. In $B F$ theory $B$ is a one form on the three dimensional manifold taking values in $\mathcal{L}_{G}^{*}$ and $F$ is a curvature two form on the manifold taking values in $\mathcal{L}_{G}$, where $\mathcal{L}_{G}^{*}$ is the dual algebra of $\mathcal{L}_{G}$. In the present case $\mathcal{L}_{G}^{*} \oplus \mathcal{L}_{G}$ coincides with the Lie algebra of $I S O(3)$ group. Due to the algebraic 


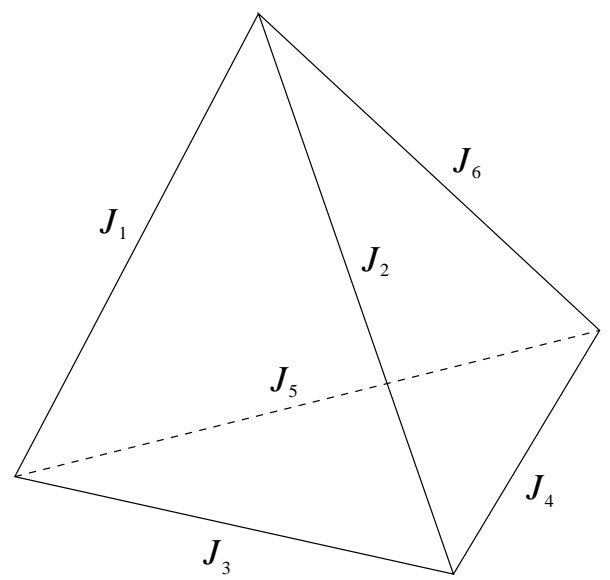

Figure 1: tetrahedron with angular momenta on the links

dual nature of $B$ and $F$, the gauge transformations of the Palatini action include the same gauge transformations (2.7) and (2.8) as ISO(3) Chern-Simons gauge theory.

\subsection{Ponzano-Regge Model}

Ponzano and Regge noticed that angular momenta of $6-j$ symbol can be identified as link lengths of a tetrahedron. In particular they showed the following approximate relation:

$$
(-1)^{\sum_{i=1}^{6} J_{i}}\left\{\begin{array}{ccc}
J_{1} & J_{2} & J_{3} \\
J_{4} & J_{5} & J_{6}
\end{array}\right\} \sim \frac{1}{\sqrt{12 \pi V}} \cos \left(S_{\text {Regge }}+\frac{\pi}{4}\right) \quad\left(\text { all } J_{i} \gg 1\right)
$$

where $S_{\text {Regge }}$ is the Regge action of Regge calculus 14] for a tetrahedron having link length $J_{k}(k=1 \sim 6)$ which correspond to the angular momentum of the corresponding 6- $j$ symbol and $V$ is the volume of the tetrahedron. Based on this observation they proposed the following partition function:

$$
Z_{\mathrm{PR}}=\lim _{\lambda \rightarrow \infty} \sum_{J \leq \lambda} \prod_{\text {vertices }} \Lambda(\lambda)^{-1} \prod_{\text {edges }}(2 J+1) \prod_{\text {tetrahedra }}(-1)^{\sum J_{i}}\left\{\begin{array}{lll}
J_{1} & J_{2} & J_{3} \\
J_{4} & J_{5} & J_{6}
\end{array}\right\} .
$$

Thus the partition function $Z_{\mathrm{PR}}$ is the product of the partition function of each tetrahedron which reproduces the cosine of the Regge action in contrast with the exponential of the Regge action in Regge calculus. There is an argument about the origin of the cosine, that right and left handed contributions of the general 
coordinate frames contribute separately and thus the summation of the exponential with the different sign factor for the Regge action appears. It is thus natural to expect that this action leads to a gravity action.

Important characteristic of the Ponzano-Regge action is that it has a topological nature on a simplicial manifold. The action is invariant under the following 2-3 and 1-4 Alexander moves. The 2-3 and 1-4 moves are related to the following $6-j$ relations:

$$
\begin{aligned}
& \sum_{K}(-1)^{K+\sum_{i=1}^{9}} J_{i}(2 K+1)\left\{\begin{array}{lll}
J_{1} & J_{8} & K \\
J_{7} & J_{2} & J_{3}
\end{array}\right\}\left\{\begin{array}{lll}
J_{7} & J_{2} & K \\
J_{6} & J_{9} & J_{4}
\end{array}\right\}\left\{\begin{array}{lll}
J_{6} & J_{9} & K \\
J_{8} & J_{1} & J_{5}
\end{array}\right\} \\
= & \left\{\begin{array}{lll}
J_{3} & J_{4} & J_{5} \\
J_{6} & J_{1} & J_{2}
\end{array}\right\}\left\{\begin{array}{lll}
J_{3} & J_{4} & J_{5} \\
J_{9} & J_{8} & J_{7}
\end{array}\right\},
\end{aligned}
$$

and

$$
\begin{aligned}
\sum_{K_{i}}\left[\prod_{i=1}^{4}\left(2 K_{i}+1\right)\right](-1)^{\sum K_{i}} \Lambda(\lambda)^{-1}\left\{\begin{array}{ccc}
J_{1} & J_{2} & J_{3} \\
K_{1} & K_{2} & K_{3}
\end{array}\right\}\left\{\begin{array}{ccc}
J_{4} & J_{6} & J_{2} \\
K_{3} & K_{1} & K_{4}
\end{array}\right\} \\
\times\left\{\begin{array}{ccc}
J_{3} & J_{4} & J_{5} \\
K_{4} & K_{2} & K_{1}
\end{array}\right\}\left\{\begin{array}{ccc}
J_{1} & J_{5} & J_{6} \\
K_{4} & K_{3} & K_{2}
\end{array}\right\}=(-1)^{\sum J_{i}}\left\{\begin{array}{lll}
J_{1} & J_{2} & J_{3} \\
J_{4} & J_{5} & J_{6}
\end{array}\right\} .
\end{aligned}
$$

The geometrical correspondence of 2-3 and 1-4 moves with two tetrahedra into three tetrahedra and one tetrahedron into four tetrahedra is obvious from Fig.2. In the formula of 1-4 move there appears the following infinite sum which is then introduced as a regularization factor in the denominator with a cutoff $\lambda$ :

$$
\begin{aligned}
\Lambda(\lambda)= & \frac{1}{2 J_{1}+1} \sum_{\substack{K_{2}, K_{3} \leq \lambda,\left|K_{2}-K_{3}\right| \leq J_{1} \leq K_{2}+K_{3}}}\left(2 K_{2}+1\right)\left(2 K_{3}+1\right) \\
= & \sum_{J=0}^{\lambda}(2 J+1)^{2} \sim \frac{4 \lambda^{3}}{3}(\lambda \rightarrow \infty) .
\end{aligned}
$$

It is known that these two Alexander moves reproduce any three dimensional simplicial manifold. Thus the partition function $Z_{\mathrm{PR}}$ is invariant under the variation of metric and is expected to be topological.

In this paper we show that the continuum limit of the lattice Ponzano-Regge model leads to the Chern-Simons gravity by explicitly constructing lattice gauge gravity model. 

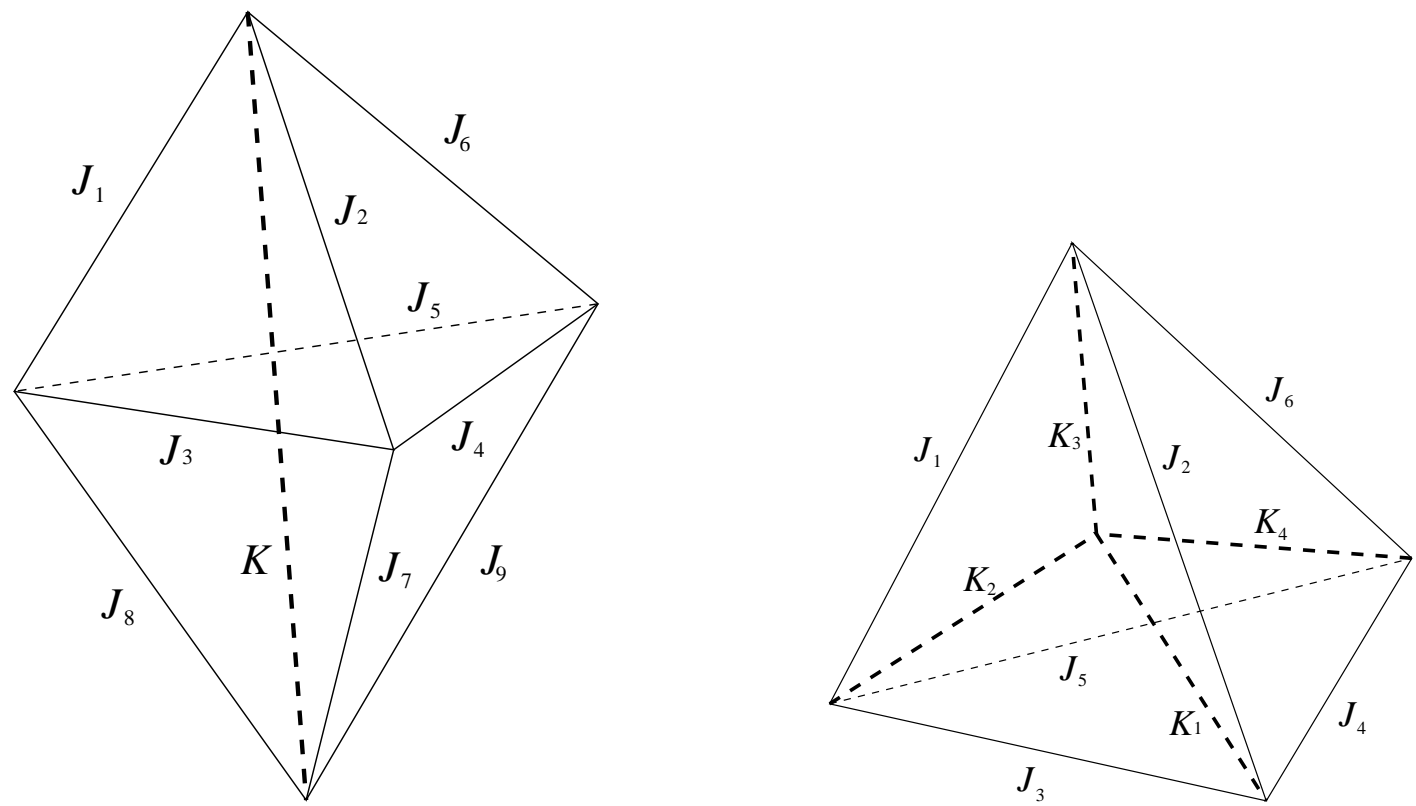

Figure 2: 2-3 move and 1-4 move

\section{Lattice Chern-Simons Gravity Action}

We consider a three-dimensional piece-wise linear simplicial manifold which is composed of tetrahedra. In 3-dimensional Regge calculus curvature is concentrated on the links of tetrahedra. We intend to formulate a lattice gravity theory in terms of gauge variables, dreibein $e$ and spin connection $\omega$. In analogy with the lattice gauge theory where link variables surrounding a plaquette induce a gauge curvature, it was proposed in [9] and [10] that dual link variables $U(\tilde{l})=e^{\omega(\tilde{l})}$ located at the boundary of a dual plaquette $\tilde{P}(\tilde{l} \in \partial \tilde{P}(l))$ associated to an original link $l$ induce the curvature of the gravity theory. It was further pointed out that the dreibein $e^{a}(l)$ is located on the original link $l$.

We propose to use a lattice version of Chern-Simons action, which is a modified version of the one in [9], and show that the length of dreibein $e(l)$ is naturally discretized. In the Chern-Simons formulation, the dreibein $e^{a}$ and spin connection $\omega^{a b}$ are Lie algebra valued gauge fields. For a moment we consider a Euclidean version of three-dimensional local Lorentz group $S O(3)$ and discuss $S U(2)$ case 


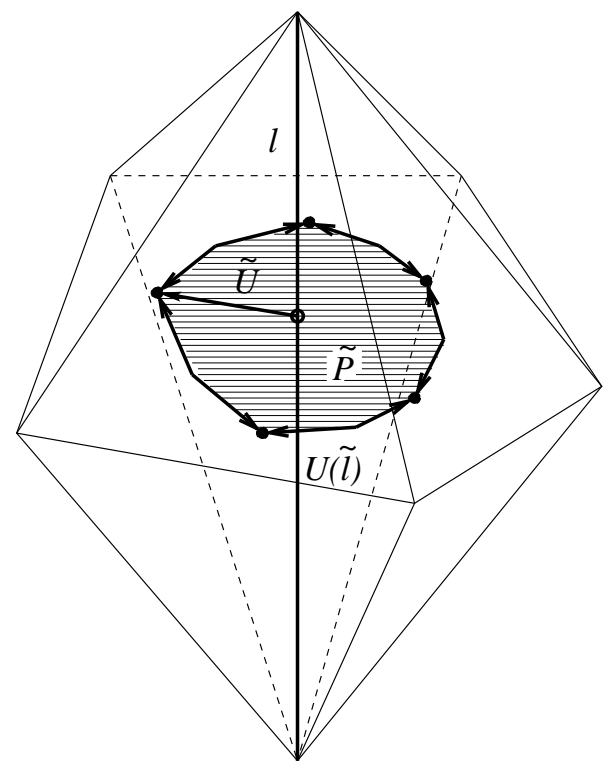

Figure 3: dual link variables on $\partial \tilde{P}$

later.

Here we slightly modify the formulation given above in order that each tetrahedron gets independent contribution to the partition function and at the same time the orientability could be naturally accommodated. We divide the dual link, which connects the centers of neighbouring tetrahedra, into two links by the center of mass of the common triangle of the neighbouring tetrahedra. We may keep to use the terminology of dual plaquette and dual link even for those modified plaquettes and links. Correspondingly we put different link variables $U$ for the doubled dual links. We then assign the directions of $U$-links inward for each tetrahedron as shown in Fig. 3 .

Using these variables, we consider the following lattice version of Chern-Simons action on the simplicial manifold,

$$
S_{\mathrm{LCS}}=\sum_{l} \epsilon_{a b c} e^{a}(l)[\ln \underset{\partial \tilde{P}(l)}{\mathbb{P}} U]^{b c}
$$

where, $\partial \tilde{P}(l)$ is a boundary of the $\tilde{P}(l)$, which is a (dual) plaquette around the link $l$, and $\underset{\partial \tilde{P}(l)}{\mathbb{1}} U$ denotes the product of $U(\tilde{l})$ along $\partial \tilde{P}(l)$. We define the "curvature" 
$F^{a b}(l)$ of the link $l$ by the following equation,

$$
\left[\prod_{\partial \tilde{P}(l)} U\right]^{a b} \equiv\left[e^{F(l)}\right]^{a b}
$$

The leading term of $F$ with respect to the lattice unit is the ordinary curvature $d \omega+\omega \wedge \omega$ similar to the ordinary lattice gauge theory.

It is the unusual point of this action (3.1) that we have taken logarithm of the Wilson loop $U$ along $\partial \tilde{P}(l)$ to extract the curvature. Because of this logarithm, however, this action has several nice features. Firstly, this action itself is already hermitian in contrast with the ordinary lattice gauge theory due to the hermiticity of Lie algebra valued curvature. Secondly, as we show in the following, the length of dreibein $e$ is discretized as a natural consequence of the logarithm.

Classically the Chern-Simons action impose a torsion free condition as an equation of motion. The torsion free nature is lost at the quantum level since we integrate out the dreibein and spin connection. We now introduce the following vanishing holonomy constraint which relates the dreibein and spin connection even at the quantum level:

$$
[\underset{\partial \tilde{P}(l)}{\mathbb{D}} U]^{a b} e^{b}=e^{a} .
$$

The dreibein $e^{a}$ associated to a original link may be parallel transported around the boundary of the dual plaquette $\partial \tilde{P}(l)$ to the original location and yet the direction of the dreibein should not be changed. We may interpret this constraint as a gauge fixing condition of gauge diffeomorphism symmetry which we will explain later. Due to the constraint the group $S O(3)$ becomes "effectively abelian", i.e. the direction of the rotation associated with the curvature is parallel to that of $e^{a}$. This can be seen as follows: we can reduce the above constraint to the following one:

$$
F^{a b} e^{b}=0
$$

hence $F^{a} \equiv \frac{1}{2} \epsilon^{a b c} F^{b c}$ is parallel to $e^{a}: e^{a} \propto F^{a}$.

Here we should reconsider the constraint (3.3). Firstly it should be noted that the $\left[\mathbb{\prod}_{\partial \tilde{P}(l)} U\right]^{a b}$ is an element of $S O(3)$ and thus the eigenvalue equation of this element always has eigenvalue +1 . Thus the number of the independent constraints 
in eq. 3.3) is not three but two. Taking into account the parallel and anti-parallel nature of $e^{a}$ and $F^{a}$ in the constraint, we can rewrite the correct constraint equation

$$
\frac{e^{3}}{|e|}\left[\prod_{a=1}^{2} \delta\left(\frac{F^{a}}{|F|}+\frac{e^{a}}{|e|}\right)+\prod_{a=1}^{2} \delta\left(\frac{F^{a}}{|F|}-\frac{e^{a}}{|e|}\right)\right],
$$

where $|e|$ and $|F|$ are length of $e^{a}$ and $F^{a}$, respectively. The coefficient factor $\frac{e^{3}}{|e|}$ is necessary to keep the rotational invariance of the constraint relation, which can be easily checked by polar coordinate expression of the constraint relation.

Now we show that discreteness of the length of the dreibein $|e|$ comes out as a natural consequence of the specific choice of the lattice gauge gravity action. We first introduce the following normalized matrix $I$,

$$
I \equiv I^{a} J_{a}, \quad I^{a} \equiv \frac{F^{a}}{\sqrt{F^{a} F_{a}}},
$$

here $\left[J_{a}\right]_{b c}=i \epsilon_{a b c}$ is the generator of $S O(3)$. This matrix satisfies the following relation,

$$
e^{i \theta I}=1-I^{2}(1-\cos \theta)+i I \sin \theta
$$

then

$$
e^{i 2 \pi n I}=1, \quad n \in \mathrm{Z} .
$$

Using the above relation and $F^{a} \propto e^{a}$ by the constraint (3.3), we find that our lattice Chern-Simons action $S_{\text {LCS }}$ has the following ambiguity:

$$
\begin{aligned}
S_{\mathrm{LCS}} & =\sum_{l} \epsilon_{a b c} e^{a}(l)\left[\ln e^{F(l)}\right]^{b c} \\
& =\sum_{l} \epsilon_{a b c} e^{a}(l)\left[\ln e^{F(l)+i 2 \pi n I}\right]^{b c} \\
& =\sum_{l}\left[2 e^{a}(l) F_{a}(l)+4 \pi n|e(l)|\right] \\
& =S_{\mathrm{LCS}}+\sum_{l} 4 \pi n|e(l)|,
\end{aligned}
$$

here $|e|$ is the length of $e^{a},|e| \equiv \sqrt{e_{a} e^{a}}$. This ambiguity leads to an ambiguity in the partition function

$$
Z=\int \mathcal{D} U \mathcal{D} e e^{i S_{\mathrm{LCS}}}=\int \mathcal{D} U \mathcal{D} e e^{i S_{\mathrm{LCS}}+i \sum_{l} 4 \pi n|e|}
$$


Imposing the single valuedness of $e^{i S_{\mathrm{LCS}}}$, we obtain the constraint that $\sum_{l} 2|e(l)|$ should be integer, or equivalently $|e(l)|$ should be half integer.

In the above arguments we have restricted the dual link variables to $S O(3)$. If we extend the arguments to $S U(2)$ the discrete nature of the dreibein is modified as follows. First of all we need to use the triplet representation of $S U(2)$ for the dual link variables since the suffix of the color variable should vary from 1 to 3 to be compatible with our lattice Chern-Simons action (3.1). We may then use the same generators of $S O(3),\left[J_{a}\right]_{b c}=i \epsilon_{a b c}$, for the triplet representation of $S U(2)$. This representation is, however, not faithful (injective). In other words an element of the triplet representation used by those generators and the corresponding element of $S U(2)$ is not one to one but one to two correspondent. Due to this degeneracy of the representation the periodicity relation (3.8) for $S U(2)$ should be modified to

$$
e^{i 4 \pi n I}=1, \quad n \in \mathrm{Z} .
$$

Accordingly we need to modify factor 2 in the corresponding relations in the above, i.e., $4|e(l)|$ should be substituted for $2|e(l)|$ in $S U(2)$ case.

\section{Gauge Invariance on the Lattice}

The gauge transformations of the continuum Chern-Simons gravity have been given by (2.6) which includes the local Lorentz gauge transformation (2.7) and the gauge transformation of diffeomorphism (2.8). We first note that the dreibein and the curvature defined in (2.5) transform adjointly under the local Lorentz gauge transformation

$$
\begin{aligned}
\delta e_{\mu}^{a} & =-\epsilon^{a b c} e_{\mu b} \tau_{c}, \\
\delta F_{\mu \nu}^{a} & =-\epsilon^{a b c} F_{\mu \nu}^{b} \tau_{c} .
\end{aligned}
$$

We consider the lattice version of the local Lorentz gauge parameters are sitting on the dual sites and the middle of the original links, the same point of the dreibein. For simplicity we consider here in this section that the dual link is not divided into two dual links by the center of original triangle. Then the dual link variable $U(\tilde{l})=e^{\omega(\tilde{l})}$ transforms under the lattice local Lorentz transformation as

$$
U(\tilde{l}) \rightarrow V^{-1} U(\tilde{l}) V^{\prime}
$$


where the gauge parameters $V$ and $V^{\prime}$ are elements of $S O(3)$ and located at the end points of dual link $\tilde{l}$. Defining the matrix form of the dreibein by $E_{\mu}^{c b}(l)=\epsilon^{a b c} e_{a \mu}(l)$, we can rewrite the lattice Chern-Simons action (3.1) by

$$
S_{\mathrm{LCS}}=\sum_{l} \operatorname{Tr}(E(l) F(l))
$$

where $F(l)^{a b}=[\ln \underset{\partial \tilde{P}(l)}{\mathbb{Q}} U]^{a b}$.

Corresponding to the continuum local Lorentz transformation, we can define the lattice version of local Lorentz transformation of $E(l)$ and $F(l)$ according to (4.2)

$$
\begin{aligned}
& E(l) \rightarrow V^{-1} E(l) V, \\
& F(l) \rightarrow V^{-1} F(l) V .
\end{aligned}
$$

It is obvious that the lattice Chern-Simons action (4.3) is invariant under the lattice local Lorentz transformation.

There are, however, some subtleties on the gauge invariance of the lattice action. In defining the lattice curvature $F(l)^{a b}=[\ln \underset{\partial \tilde{P}(l)}{\mathbb{1}} U]^{a b}$, we need to choose a starting

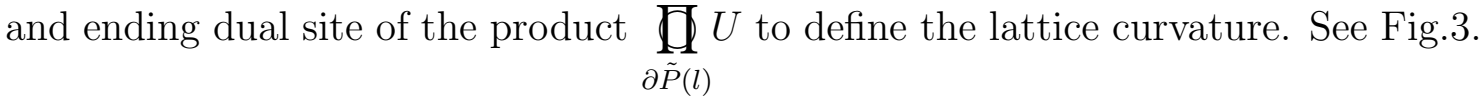
We then need to bridge between the dual site and the center of the original link of $e(l)$ by new link variables $\tilde{U}$ and $\tilde{U}^{-1}$. The action associated with this particular dual plaquette is

$$
\begin{aligned}
\operatorname{Tr}\left(E(l) \tilde{U}\left[\ln \prod_{\partial \tilde{P}(l)} U\right] \tilde{U}^{-1}\right) & =\operatorname{Tr}\left(V^{-1} E(l) V V^{-1} \tilde{U} V^{\prime} V^{\prime-1}\left[\ln \underset{\partial \tilde{P}(l)}{\prod} U\right] V^{\prime} V^{\prime-1} \tilde{U}^{-1} V\right) \\
& \rightarrow \operatorname{Tr}\left(E^{\prime}(l)\left[\ln \prod_{\partial \tilde{P}(l)} U^{\prime}\right]\right) .
\end{aligned}
$$

Then the newly introduced link variable transforms similar as the dual link variable $\tilde{U} \rightarrow V^{-1} \tilde{U} V^{\prime}$, where $V$ and $V^{\prime}$ are located on the the center of the original link and the dual site, respectively. We can, however, use one of the gauge parameters, say the one at center of the original link, $V^{-1}$, to tune in such a way that this link variable leads to a trivial factor $\tilde{U} \rightarrow V^{-1} \tilde{U} V^{\prime} \rightarrow 1$. We then redefine the matrix form of the dreibein $V^{-1} E(l) V=E^{\prime}(l)$ and the curvature $V^{\prime-1}\left[\ln \underset{\partial \tilde{P}(l)}{\prod} U\right] V^{\prime}=\ln \prod_{\partial \tilde{P}(l)} U^{\prime}$. We can 


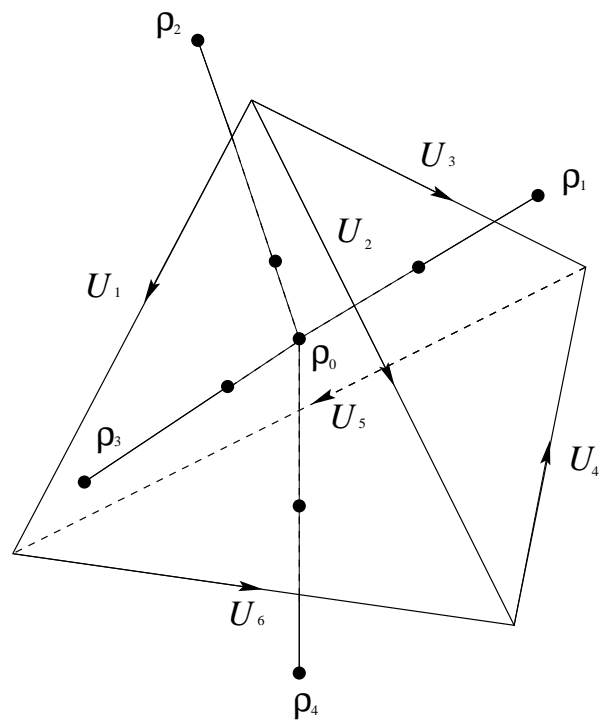

Figure 4: dual tetrahedron : $\rho_{k}(k=0 \sim 4)$ at original site, $U_{k}(k=1 \sim 4)$ on dual link

thus gauge away the variable $\tilde{U}$. This can be allowed since we have enough gauge parameters on the dual sites and on the original links because of the geometrical reason that our simplicial manifold is constructed out of tetrahedra. We can now identify the final expression of (4.5) as the one in (4.3). Accepting this arguments we have confirmed that our lattice Chern-Simons action is invariant under lattice local Lorentz transformation.

The continuum Chern-Simons gravity action is invariant under the gauge transformation of diffeomorphism (2.8) which transforms dreibein $e_{\mu}^{a}$ but not spin connection $\omega_{\mu}^{a}$. We try to identify the lattice counter part of this gauge transformation and show the gauge invariance. The gauge invariance of the continuum action by the gauge diffeomorphism (2.8) can be shown by using Bianchi identity. Here we first formulate the lattice version of integrated Bianchi identity

$$
\int_{M} D F=\int_{\partial M} F+\int_{M}[\omega, F]=0
$$

For a three dimensional simplicial manifold randomly constructed from tetrahedra, several original links could be connected to an original site. Since an original link $l$ is dual to the corresponding dual plaquette $\tilde{P}(l)$, an original site is surrounded 
by dual plaquettes which topologically construct $S^{2}$ sphere. In general the sphere could take arbitrary shape. For simplicity we here assume that the sphere is a tetrahedron. In this case the original site is in the center of the tetrahedron and the triangles of the tetrahedron are the dual plaquettes. Dual link variables $U_{k}$ ( $k=1 \sim 6$ ) are located on the dual links, the edges of the tetrahedron, where the directions of the dual link variables can be arbitrarily chosen. Original links $l_{k}$ $(k=1 \sim 4)$ are sticking from the center through the triangles. See Fig: 4 .

We first note the following identity:

$$
\prod U \equiv U_{2} U_{4} U_{3}^{-1} U_{3} U_{5} U_{1}^{-1} U_{1} U_{6} U_{2}^{-1} U_{2} U_{6}^{-1} U_{5}^{-1} U_{4}^{-1} U_{2}^{-1}=1
$$

We now define

$$
\begin{aligned}
& \ln \left(U_{2} U_{4} U_{3}^{-1}\right)=F_{1}, \quad \ln \left(U_{3} U_{5} U_{1}^{-1}\right)=F_{2}, \\
& \ln \left(U_{1} U_{6} U_{2}^{-1}\right)=F_{3}, \quad \ln \left(U_{2} U_{6}^{-1} U_{5}^{-1} U_{4}^{-1} U_{2}^{-1}\right)=F_{4}^{\prime} .
\end{aligned}
$$

Due to the Baker-Hausdorff formula the above relations lead

$$
0=\ln \left(\prod U\right)=\sum_{k=1}^{3} F_{k}+F_{4}^{\prime}+\frac{1}{2} \sum_{\substack{i<j \\ i, j=1,2,3}}\left[F_{i}, F_{j}\right]+\frac{1}{2} \sum_{k=1}^{3}\left[F_{k}, F_{4}^{\prime}\right]+\cdots
$$

On the other hand the lattice version of Bianchi identity (4.6) can be written

$$
\sum_{k=1}^{3} F_{k}+F_{4}^{\prime}+\sum_{k=1}^{3}\left[\Omega_{k}, F_{k}\right]+\left[\Omega_{4}, F_{4}^{\prime}\right]=0
$$

Here we have defined irregular lattice curvature $F_{4}^{\prime}$ which has $U_{2}$ and $U_{2}^{-1}$ in the product of dual link variables. This type of irregular definition is unavoidable in deriving lattice Bianchi identity due to the geometrical reason leading to the type of identity of (4.7). We can then identify

$$
\begin{array}{ll}
\Omega_{1}=-\frac{1}{4}\left(F_{2}+F_{3}+F_{4}^{\prime}\right)+\cdots, & \Omega_{2}=-\frac{1}{4}\left(-F_{1}+F_{3}+F_{4}^{\prime}\right)+\cdots, \\
\Omega_{3}=-\frac{1}{4}\left(-F_{1}-F_{2}+F_{4}^{\prime}\right)+\cdots, & \Omega_{4}=-\frac{1}{4}\left(-F_{1}-F_{2}-F_{3}\right)+\cdots .
\end{array}
$$

In comparing the continuum Bianchi identity (4.6) with the lattice version (4.10), we notice that $\left[\Omega_{k}, F_{k}\right]$ term is the integrand in the volume integration. $\Omega_{k}$ should 
thus be defined as an average spin connection inside the dual volume, the tetrahedron in the present case. Since the curvature itself could be interpreted as an average spin connection on a dual plaquette, the $\Omega_{k}$ defined in (4.11) is the particular average of the curvature and thus can be interpreted as the average spin connection in the dual volume with respect to $F_{k}$.

We now show the lattice gauge diffeomorphism invariance of the lattice ChernSimons action (4.3). We first consider the term related to the fourth link $l_{4}$ and make local Lorentz transformation

$$
\begin{aligned}
\operatorname{Tr}\left(E\left(l_{4}\right) F\left(l_{4}\right)\right) & =\operatorname{Tr}\left(V^{-1} E\left(l_{4}\right) V V^{-1} F\left(l_{4}\right) V\right) \\
& \rightarrow \operatorname{Tr}\left(E^{\prime}\left(l_{4}\right) U_{2} F\left(l_{4}\right) U_{2}^{-1}\right)=\operatorname{Tr}\left(E^{\prime}\left(l_{4}\right) F^{\prime}\left(l_{4}\right)\right),
\end{aligned}
$$

where $F\left(l_{4}\right) \equiv F_{4}=\ln \left(U_{6}^{-1} U_{5}^{-1} U_{4}^{-1}\right)$. Here we take the gauge choice $V=U_{2}^{-1}$ and further redefine $V^{-1} E\left(l_{4}\right) V=E^{\prime}\left(l_{4}\right)$, we obtain the final expression. From now on we identify $E^{\prime}\left(l_{4}\right)=E\left(l_{4}\right)$. In this way we can introduce the unusual definition of the curvature $F_{4}^{\prime}$ of (4.8) in the lattice Chern-Simons action. Hereafter we rename $F_{4}^{\prime}$ as $F_{4}$.

The lattice version of the gauge transformation of diffeomorphism (2.8) can be given by using the $\Omega_{k}$ defined above

$$
\delta E_{k}\left(l_{k}\right)=-\rho_{k}+\rho_{0}-\left[\Omega_{k}, \rho_{0}\right],
$$

where $\rho_{k}$ is the matrix gauge parameter, $\left(\rho_{k}\right)^{a b} \equiv \epsilon^{b a c} \rho_{k}^{c}$.

Then the lattice gauge transformation of diffeomorphism for the lattice action (4.3) leads

$$
\begin{aligned}
\delta S_{\mathrm{LCS}} & =\sum_{l} \operatorname{Tr}(\delta E(l) F(l)) \\
& =\sum_{k} \operatorname{Tr}\left\{\left(-\rho_{k}+\rho_{0}-\left[\Omega_{k}, \rho_{0}\right]\right) F_{k}\right\}+\cdots \\
& =\sum_{k} \operatorname{Tr}\left\{\rho_{0}\left(\sum_{k=1}^{4} F_{k}+\sum_{k=1}^{4}\left[\Omega_{k}, F_{k}\right]\right)\right\}+\cdots \\
& =0
\end{aligned}
$$

due to the Bianchi identity (4.10). Here we have used the following relation:

$$
\operatorname{Tr}\left(\left[\Omega_{k}, \rho_{0}\right] F_{k}\right)=-\operatorname{Tr}\left(\rho_{0}\left[\Omega_{k}, F_{k}\right]\right) .
$$


We have thus completed the proof of the invariance of the lattice Chern-Simons action under the lattice gauge transformation of diffeomorphism.

We now point out that the constraint (3.3) or equivalently (3.4) breaks the lattice gauge diffeomorphism while the lattice Chern-Simons action itself is invariant, as is shown above. The lattice dreibein is transformed but the lattice curvature is not transformed under the lattice gauge transformation of the diffeomorphism. The precise expression of the constraint (3.5) tells us that the dreibein $e^{a}$ can be rotated by using two gauge parameters of the gauge transformation of diffeomorphism to be parallel or anti-parallel to the curvature $F^{a}$. The length of the dreibein is discretized and thus the third gauge parameter can be exhausted. In this sense we can identify the equivalent constraint, (3.3), (3.4), (3.5) as a gauge fixing condition of the lattice gauge transformation of diffeomorphism.

\section{Calculation of Partition Function}

In the previous section we have found that the length of dreibein is discretized to half integer for $S O(3)$ and a quarter for $S U(2)$. To be specific we restrict our arguments for $S O(3)$ for a moment. In order to accommodate the discrete nature of the length of the dreibein, we first note an identity

$$
\int_{\left|e_{i}\right|}^{\left|e_{f}\right|} d|e|=\int_{\left|e_{i}\right|}^{\left|e_{f}\right|} \frac{1}{2} \sum_{J=0}^{\infty} \delta\left(|e|-\frac{J}{2}\right) d|e|
$$

where $\left|e_{f}\right|$ and $\left|e_{i}\right|$ are half integer. We can thus safely insert the delta function constraints without changing the value of the partition function.

Then the total partition function is

$$
\begin{aligned}
Z=\int \mathcal{D} U & \prod_{l} Z_{l} \\
Z_{l}=\int d^{3} e & \frac{e^{3}}{|e|}\left[\prod_{a=1}^{2} \delta\left(\frac{F^{a}}{|F|}+\frac{e^{a}}{|e|}\right)+\prod_{a=1}^{2} \delta\left(\frac{F^{a}}{|F|}-\frac{e^{a}}{|e|}\right)\right] \\
& \times \frac{1}{2} \sum_{J=0}^{\infty} \delta\left(|e|-\frac{J}{2}\right) e^{2 i e^{a} F^{a}}
\end{aligned}
$$

where $Z_{l}$ is the partition function associated with a link $l$. 


\section{$5.1 \quad e$ integration}

Due to the rotational invariance of the constraints, we can take $e^{3}$ as the third direction of local Lorentz frame without loss of generality. We can then evaluate $e^{a}$ integral of $Z_{l}$ immediately thanks to the delta functions

$$
\begin{aligned}
Z_{l} & =\int d^{3} e|e|^{2} \frac{e^{3}}{|e|}\left[\prod_{a=1}^{2} \delta\left(e^{a}+|e| \frac{F^{a}}{|F|}\right)+\prod_{a=1}^{2} \delta\left(e^{a}-|e| \frac{F^{a}}{|F|}\right)\right] \frac{1}{2} \sum_{J} \delta\left(|e|-\frac{J}{2}\right) e^{2 i e^{a} F^{a}} \\
& =\frac{1}{2} \sum_{J}\left(\frac{J}{2}\right)^{2}\left(e^{2 i \frac{J}{2}|F|}+e^{-2 i \frac{J}{2}|F|}\right) \\
& =\sum_{J} \frac{1}{4} J^{2} \cos (J|F|) .
\end{aligned}
$$

Using the following formula for the character $\chi_{J}$ of the spin- $J$ representation of $S O(3)$

$$
\chi_{J}\left(e^{i \theta^{a} J_{a}}\right)=\chi_{J}(|\theta|)=\frac{\sin \left((2 J+1) \frac{|\theta|}{2}\right)}{\sin \left(\frac{|\theta|}{2}\right)},
$$

where $|\theta|$ is the length of $\theta^{a}$, we find

$$
\chi_{J}(|F|)-\chi_{J-1}(|F|)=2 \cos (J|F|) .
$$

Hence we can naively calculate the link partition function,

$$
\begin{aligned}
Z_{l} & =\sum_{J=1}^{\infty} \frac{1}{8} J^{2}\left(\chi_{J}-\chi_{J-1}\right) \\
& =\frac{1}{8}\left[\sum_{J=0}^{\infty} J^{2} \chi_{J}-\sum_{J=0}^{\infty}(J+1)^{2} \chi_{J}\right] \\
& =\frac{1}{8} \sum_{J=0}^{\infty}\left[J^{2}-(J+1)^{2}\right] \chi_{J} \\
& =-\frac{1}{8} \sum_{J=0}^{\infty}(2 J+1) \chi_{J} .
\end{aligned}
$$

This calculation is not precise, because the summation is not convergent. We need to show that there is a regularization procedure which leads to a validity of the above calculation after the regularization.

We propose to use the heat kernel regularization. We first consider the following heat equation:

$$
\triangle K\left(U, U^{\prime} ; t\right)=\frac{\partial}{\partial t} K\left(U, U^{\prime} ; t\right), \quad \lim _{t \rightarrow 0} K\left(U, U^{\prime} ; t\right)=\delta\left(U, U^{\prime}\right),
$$


where the laplacian is defined on the group manifold, $K\left(U, U^{\prime} ; t\right)$ is the heat kernel and $\delta\left(U, U^{\prime}\right)$ is the delta function. Then the regularized character will be given by

$$
\chi_{J}(U ; t)=\int d U^{\prime} \chi_{J}\left(U^{\prime}\right) K\left(U, U^{\prime} ; t\right)
$$

All these quantities are defined on the group manifold $S O(3)$. In particular the laplacian on the group manifold will be related in general to the 2nd Casimir operator, the square of the angular momentum in case of $S O(3)$. Hence the character of spin- $J$ representation $\chi_{J}$, which is essentially the trace of the matrix representation, is the eigenfunction of the laplacian with the eigen value $-J(J+1)$,

$$
\triangle \chi_{J}=-J(J+1) \chi_{J}
$$

Noting the completeness of the character

$$
\delta\left(U, U^{\prime}\right)=\sum_{J} \chi_{J}(U) \chi_{J}\left(U^{\prime}\right)
$$

we can immediately obtain the heat kernel solution

$$
K\left(U, U^{\prime} ; t\right)=\sum_{J} e^{-J(J+1) t} \chi_{J}(U) \chi_{J}\left(U^{\prime}\right)
$$

Substituting the heat kernel solution into (5.7) and using the orthogonality of the character,

$$
\int d U \chi_{I}(U) \chi_{J}(U)=\delta_{I J}
$$

we obtain an explicit form of regularized character

$$
\chi_{J}(U ; t)=e^{-J(J+1) t} \chi_{J}(U) .
$$

The summation is now convergent and should be replaced by

$$
\begin{aligned}
Z_{l} & =\sum_{J} \frac{1}{8} J^{2}\left(\chi_{J}-\chi_{J-1}\right) \\
& \rightarrow \sum_{J} \frac{1}{8} J^{2}\left(\chi_{J} e^{-J(J+1) t}-\chi_{J-1} e^{-(J-1) J t}\right),
\end{aligned}
$$

which leads to the regularized result

$$
Z_{l}=-\frac{1}{8} \sum_{J}(2 J+1) \chi_{J} e^{-J(J+1) t} .
$$


It is interesting to note that this link partition function coincides with the heatkernel lattice gauge theory action but the interpretations and the origin of the terms are quite different [6] [7] [15]. This regularization factor $e^{-J(J+1) t}$ breaks the Alexander invariance of the partition function but it will be recovered at the end of the calculation when we take the limit $t \rightarrow 0$.

One of the remarkable points of this result is that the factor $2 J+1$ has appeared in the link partition function $Z_{l}$, which is the same and necessary factor for the P-R model to assure the Alexander invariance. Another important point is that the character has appeared after the $e^{a}$ integration, which makes $U$ integration straightforward.

\section{$5.2 \quad U$ integration}

After $e^{a}$ integration and dividing the unimportant constant factor $\prod_{l}(-1 / 8)$, the partition function leads

$$
Z=\int \mathcal{D} U \prod_{l} \sum_{J=0}^{\infty}(2 J+1) \chi_{J}(\underset{\partial \tilde{P}(l)}{\mathbb{1}} U) e^{-J(J+1) t}
$$

where we take $t \rightarrow 0$ limit in the end of calculation. We now carry out $D U$ integration of this partition function. Thanks to the character of the partition function, $D U$ integration is straightforward. We show that the Ponzano-Regge partition function will be reproduced after $D U$ integration with 6 - $j$ symbols together with correct coefficients and sign factors.

Before getting into the details we figure out how 6- $j$ symbols appear. The character in the partition function is a product of $D$-function around the boundary of dual plaquette associated to a original link. Each tetrahedron has six original links and there are two dual links which is a part of a product on the boundary of the dual plaquette associated to each original link. In other words three dual links associated to a $D U$ integration thrust into each triangle from the center of the tetrahedron. Therefore twelve dual links are associated to a tetrahedron. Each $D U$ integration of the product of three $D$-function reproduces two $3-j$ symbols, thus we get eight $3-j$ symbols for each tetrahedron. Four out of eight $3-j$ symbols lead to a $6-j$ symbol and the rest of four $3-j$ symbols lead to give trivial factor together with the $3-j$ 

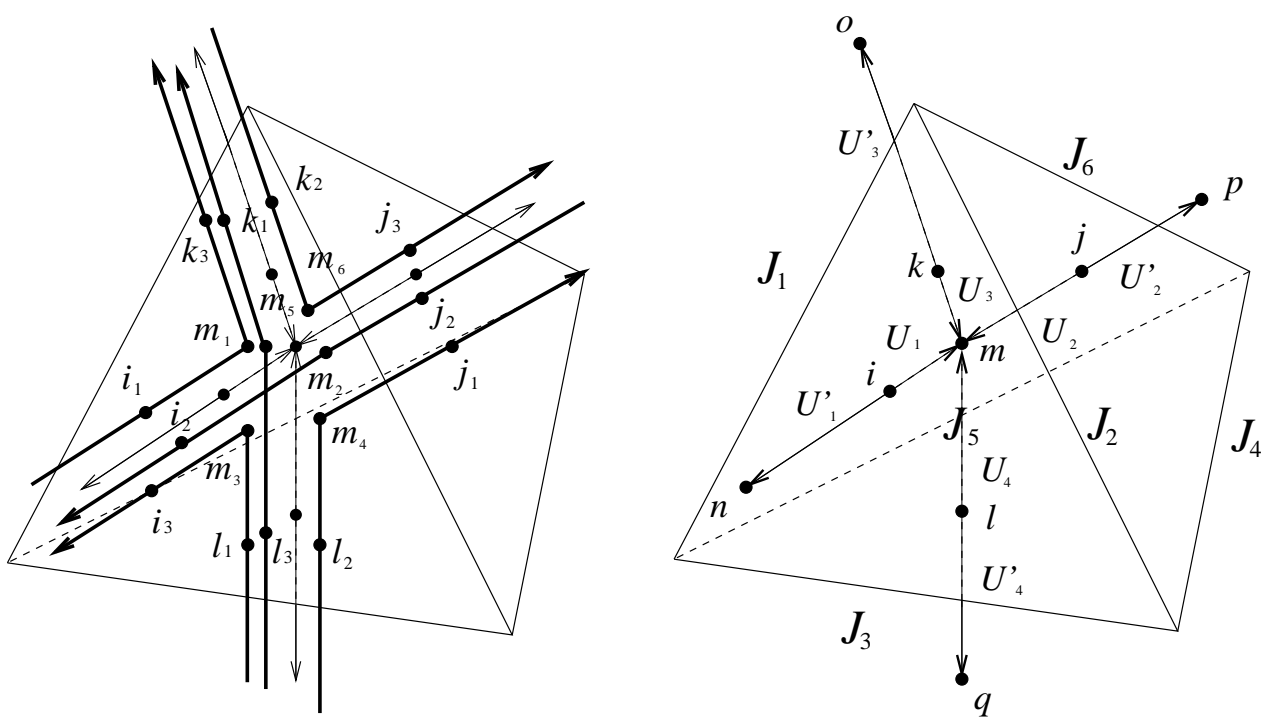

Figure 5: dual links related to neighbouring tetrahedra and the orientability

symbols from the neighbouring tetrahedra.

We first note that the character appearing in the partition function is a product of $D$-functions

$$
\chi_{J}(|F|)=\chi_{J}\left(\underset{\partial \tilde{P}(l)}{\prod} U\right)=D_{m_{1} m_{2}}^{J}\left(U_{1}\right) D_{m_{2} m_{3}}^{J}\left(U_{2}\right) \cdots D_{m_{k} m_{1}}^{J}\left(U_{k}\right),
$$

where $U_{i}$ is a dual link variables on the boundary of dual plaquette $\tilde{P}(l)$ associated to a link $l$ and $m_{i}$ is the third component of angular momentum $J$ which is assigned to the link $l$. As we have already pointed out that the direction of $U_{i}$ for each link is defined inward for each tetrahedron. On the other hand the direction of the loop composed of the product of dual links associated to the link $l$ can be chosen arbitrarily. Therefore some of $U_{i}$ in the above $D$-functions are $U_{i}^{\dagger}$. If the original link $l$ is a link of a particular tetrahedron, two $D$-functions out of the above product are located inside the tetrahedron.

We now choose a particular situation which is shown in Fig.5. The twelve $D$ functions associated to this particular tetrahedron are

$$
I_{U_{1} U_{2} U_{3} U_{4}}=\int \prod_{i=1}^{4} D U_{i} D_{i_{1} m_{1}}^{J_{1}}\left(U_{1}\right) D_{m_{1} k_{3}}^{J_{1}}\left(U_{3}^{\dagger}\right) \cdot D_{j_{2} m_{2}}^{J_{2}}\left(U_{2}\right) D_{m_{2} i_{2}}^{J_{2}}\left(U_{1}^{\dagger}\right)
$$




$$
\begin{aligned}
& \times D_{l_{1} m_{3}}^{J_{3}}\left(U_{4}\right) D_{m_{3} i_{3}}^{J_{3}}\left(U_{1}^{\dagger}\right) \cdot D_{l_{2} m_{4}}^{J_{4}}\left(U_{4}\right) D_{m_{4} j_{1}}^{J_{4}}\left(U_{2}^{\dagger}\right) \\
& \times D_{l_{3} m_{5}}^{J_{5}}\left(U_{4}\right) D_{m_{5} k_{1}}^{J_{5}}\left(U_{3}^{\dagger}\right) \cdot D_{k_{2} m_{6}}^{J_{6}}\left(U_{3}\right) D_{m_{6} j_{3}}^{J_{6}}\left(U_{2}^{\dagger}\right) .
\end{aligned}
$$

We pick up the $D$-functions associated to $D U_{1}$ integration

$$
I_{U_{1}}=(-)^{i_{2}-m_{2}+i_{3}-m_{3}} \int D U_{1} D_{i_{1} m_{1}}^{J_{1}}\left(U_{1}\right) D_{-i_{2}-m_{2}}^{J_{2}}\left(U_{1}\right) D_{-i_{3}-m_{3}}^{J_{3}}\left(U_{1}\right)
$$

where we have used the following formula to rewrite only with $U_{1}$ variable:

$$
D_{m n}^{I}\left(U^{\dagger}\right)=D_{n m}^{I *}(U)=(-)^{n-m} D_{-n-m}^{I}(U) .
$$

We can now use the formula relating the integration of three $D$-functions and two $3-j$ symbols 16,

$$
\int D U D_{m_{1} n_{1}}^{I}(U) D_{m_{2} n_{2}}^{J}(U) D_{m_{3} n_{3}}^{K}(U)=\left(\begin{array}{ccc}
I & J & K \\
m_{1} & m_{2} & m_{3}
\end{array}\right)\left(\begin{array}{ccc}
I & J & K \\
n_{1} & n_{2} & n_{3}
\end{array}\right),
$$

which leads to the result of $D U_{1}$ related integration

$$
I_{U_{1}}=(-)^{i_{2}-m_{2}+i_{3}-m_{3}}\left(\begin{array}{ccc}
J_{1} & J_{2} & J_{3} \\
m_{1} & -m_{2} & -m_{3}
\end{array}\right)\left(\begin{array}{ccc}
J_{1} & J_{2} & J_{3} \\
i_{1} & -i_{2} & -i_{3}
\end{array}\right) .
$$

After carrying out $D U_{2} D U_{3} D U_{4}$ integration, we obtain

$$
\begin{aligned}
& I_{U_{1} U_{2} U_{3} U_{4}}=(-)^{i_{2}-m_{2}+i_{3}-m_{3}}\left(\begin{array}{ccc}
J_{1} & J_{2} & J_{3} \\
m_{1} & -m_{2} & -m_{3}
\end{array}\right)\left(\begin{array}{ccc}
J_{1} & J_{2} & J_{3} \\
i_{1} & -i_{2} & -i_{3}
\end{array}\right) \\
& \times(-)^{j_{1}-m_{4}+j_{3}-m_{6}}\left(\begin{array}{ccc}
J_{4} & J_{2} & J_{6} \\
-m_{4} & m_{2} & -m_{6}
\end{array}\right)\left(\begin{array}{ccc}
J_{4} & J_{2} & J_{6} \\
-j_{1} & j_{2} & -j_{3}
\end{array}\right) \\
& \times(-)^{k_{3}-m_{1}+k_{1}-m_{5}}\left(\begin{array}{ccc}
J_{1} & J_{5} & J_{6} \\
-m_{1} & -m_{5} & m_{6}
\end{array}\right)\left(\begin{array}{ccc}
J_{1} & J_{5} & J_{6} \\
-k_{3} & -k_{1} & k_{2}
\end{array}\right) \\
& \times\left(\begin{array}{ccc}
J_{4} & J_{5} & J_{3} \\
m_{4} & m_{5} & m_{3}
\end{array}\right)\left(\begin{array}{ccc}
J_{4} & J_{5} & J_{3} \\
l_{2} & l_{3} & l_{1}
\end{array}\right) .
\end{aligned}
$$

We now use the formula,

$$
\begin{aligned}
& \left\{\begin{array}{lll}
J_{1} & J_{2} & J_{3} \\
J_{4} & J_{5} & J_{6}
\end{array}\right\}=\sum_{\text {all } m_{i}}(-1)^{\sum_{i}\left(J_{i}-m_{i}\right)}\left(\begin{array}{ccc}
J_{1} & J_{2} & J_{3} \\
-m_{1} & -m_{2} & -m_{3}
\end{array}\right) \\
& \times\left(\begin{array}{ccc}
J_{1} & J_{5} & J_{6} \\
m_{1} & -m_{5} & m_{6}
\end{array}\right)\left(\begin{array}{ccc}
J_{4} & J_{2} & J_{6} \\
m_{4} & m_{2} & -m_{6}
\end{array}\right)\left(\begin{array}{ccc}
J_{4} & J_{5} & J_{3} \\
-m_{4} & m_{5} & m_{3}
\end{array}\right),
\end{aligned}
$$


for the four $3-j$ symbols which carry $m_{i}$ suffices associated to the center of the tetrahedron. We then find 6- $j$ symbols after $D U_{1} D U_{2} D U_{3} D U_{4}$ integration

$$
\begin{aligned}
I_{U_{1} U_{2} U_{3} U_{4}} & =(-)^{\sum_{i=1}^{6} J_{i}}\left\{\begin{array}{lll}
J_{1} & J_{2} & J_{3} \\
J_{4} & J_{5} & J_{6}
\end{array}\right\} \\
& \times(-)^{i_{2}+i_{3}}\left(\begin{array}{ccc}
J_{1} & J_{2} & J_{3} \\
i_{1} & -i_{2} & -i_{3}
\end{array}\right)(-)^{j_{3}+j_{1}}\left(\begin{array}{ccc}
J_{4} & J_{2} & J_{6} \\
-j_{1} & j_{2} & -j_{3}
\end{array}\right) \\
& \times(-)^{k_{3}+k_{1}}\left(\begin{array}{ccc}
J_{1} & J_{5} & J_{6} \\
-k_{3} & -k_{1} & k_{2}
\end{array}\right)\left(\begin{array}{lll}
J_{4} & J_{5} & J_{3} \\
l_{2} & l_{3} & l_{1}
\end{array}\right) .
\end{aligned}
$$

Here we are considering $S O(3)$ case then the third component of the angular momentum $m_{i}$ is integer and thus we can use the relation $(-)^{m_{i}}=(-)^{-m_{i}}$ which is not correct if $m_{i}$ is half integer in case of $S U(2)$. We now look at the rest of the 3- $j$ symbols in eq. (5.20) which carry the suffices $i, j, k, l$. As we can see from Fig. that $D U_{1}$ integration reproduces two $3-j$ symbols and one of them associated to the suffices $m_{k}$ is absorbed to reproduce the $6-j$ symbol and the other $3-j$ carrying the suffices $i_{k}$ could be combined with another $3-j$ symbol obtained from $D U_{1}^{\prime}$ integrations of the neighbouring tetrahedron. Those $3-j$ symbols are associated to the boundary triangle of the two neighbouring tetrahedron carrying suffix $i_{k}$. In this particular case of Fig.5 we obtain the following two $3-j$ symbols

$$
I_{i}^{b}=\sum_{i_{1} i_{2} i_{3}}(-)^{i_{1}+i_{2}+i_{3}}\left(\begin{array}{ccc}
J_{1} & J_{2} & J_{3} \\
i_{1} & -i_{2} & -i_{3}
\end{array}\right)\left(\begin{array}{ccc}
J_{1} & J_{2} & J_{3} \\
i_{1} & -i_{2} & -i_{3}
\end{array}\right) .
$$

Since the three angular momentum vectors $J_{1}, J_{2}, J_{3}$ construct the boundary triangle, the third components satisfy the relation $i_{1}-i_{2}-i_{3}=0$. Using the following formula:

$$
\sum_{m_{1} m_{2} m_{3}}\left(\begin{array}{ccc}
J_{1} & J_{2} & J_{3} \\
m_{1} & m_{2} & m_{3}
\end{array}\right)\left(\begin{array}{ccc}
J_{1} & J_{2} & J_{3} \\
m_{1} & m_{2} & m_{3}
\end{array}\right)=1,
$$

and noting $(-)^{i_{1}+i_{2}+i_{3}}=(-)^{i_{1}-i_{2}-i_{3}}=1$ for $S O(3)$ case, these two $3-j$ symbols lead to a trivial factor.

It should be pointed out here that the above factor reproduces a negative sign if $i_{k}$ is half integer in case of $S U(2)$ since $(-)^{i_{1}+i_{2}+i_{3}}=(-)^{2 i_{1}}(-)^{-i_{1}+i_{2}+i_{3}}=-1$. Some of negative sign factors can be removed by using the triangle relation and $(-)^{m_{1}-i_{1}}=(-)^{-m_{1}+i_{1}}$, which holds even for half integer values since $m_{1}$ and $i_{1}$ are both the third components of $J_{1}$. We cannot, however, get rid of all the negative sign 
factors for the dual link loop associated to the original link in this way. Therefore we need to point out that there appear floating negative sign factors for $S U(2)$ case.

Finally we have found that our partition function is the same as that of the Ponzano-Regge model, except for the regularization factor $\Lambda(\lambda)=\sum_{J=0}^{\lambda}(2 J+1)^{2}$. Since our partition function is divergent with the same reason, we should introduce the same regularization factor as the P-R model.

\section{Conclusion and the Interpretations}

We have proposed the partition function of the $I S O(3)$ lattice Chern-Simons action

$$
\begin{aligned}
Z_{\mathrm{LCS}}= & \lim _{t \rightarrow 0} \lim _{\lambda \rightarrow \infty} \int \frac{\mathcal{D} U \mathcal{D} e}{\prod_{l}\left(-\frac{1}{8}\right) \prod_{\text {verticies }} \Lambda(\lambda)} \int \mathcal{D} U^{\prime} K\left(U, U^{\prime} ; t\right) \\
& \times \frac{e^{3}}{|e|}\left[\prod_{a=1}^{2} \delta\left(\frac{F^{a}}{F}+\frac{e^{a}}{e}\right)+\prod_{a=1}^{2} \delta\left(\frac{F^{a}}{F}-\frac{e^{a}}{e}\right)\right] \sum_{J=0}^{\lambda} \delta\left(e-\frac{J}{2}\right) e^{i S_{\mathrm{LCS}}\left(e, U^{\prime}\right)}
\end{aligned}
$$

which exactly coincides with the Ponzano-Regge model after the integration of the dreibein and the dual link variables. The discreteness of the length of the dreibein is the natural consequence of the logarithm form in the lattice Chern-Simons action. On the simplicial lattice manifold constructed from tetrahedra, the dreibeins are located on the original links while the lattice version of the spin connection, the dual link variables are located on the dual links. We have explicitly shown that the lattice Chern-Simons action is invariant under the lattice version of local Lorentz transformation and the lattice gauge diffeomorphism. In order to get the topological gravity theory at the quantum level, we need a constraint which solves the spin connection as a function of the dreibein. We have found the constraint which can be interpreted as the gauge fixing condition of the lattice gauge diffeomorphism.

Since the Ponzano-Regge model is invariant under the 2-3 and 1-4 Alexander moves, the partition function is invariant under how the three dimensional space is divided into small pieces by tetrahedra. It is natural to expect that the partition function is invariant in the continuum limit and the lattice Chern-Simons action leads to the continuum Chern-Simons action.

It is interesting to note that the algebraic dual nature of the one forms $e$ and $\omega$ in the $B F$ theory or equivalently in the Palatini action, is reflected on the geometric 
dual nature of $e$ and $U=\mathrm{e}^{\omega}$ on the lattice. In other words, $e$ and $\omega$ are defined in the Lie algebra $\mathcal{L}_{G}^{*}$ and $\mathcal{L}_{G}$, respectively, where $\mathcal{L}_{G}^{*}$ and $\mathcal{L}_{G}$ are dual to each other with $G=S O(3)$ and $\mathcal{L}_{G}^{*} \oplus \mathcal{L}_{G}=I S O(3)[13]$. The one form $e$ and the link variable $U=\mathrm{e}^{\omega}$ are defined on a original links and dual links, respectively, and thus the dual nature of the algebra is reflected in the geometry on the lattice.

In the $I S O(3)$ lattice Chern-Simons action there are 6 gauge parameters. Two gauge parameters of the lattice gauge diffeomorphism can be used to rotate the dreibein $e^{a}$ to be parallel or anti-parallel to the curvature $F^{a}$ and the remaining one gauge parameter of the lattice gauge diffeomorphism can be exhausted to make the length of the dreibein discrete. There remain three gauge parameters of the lattice local Lorentz gauge symmetry, which are expected to convert into the three vector parameters of general coordinate diffeomorphism symmetry. There are two reasons to expect this scenario. Firstly the lattice action coincides with the PonzanoRegge model which is Alexander move invariant and is thus expected to be metric independent. In fact the lattice Chern-Simons action in the continuum limit is metric independent since it is composed of one form. Secondly the general coordinate transformation of diffeomorphism and the local Lorentz transformation are on shell equivalent in the continuum $I S O(3)$ Chern-Simons gravity [1].

In this paper we have concentrated on the relation between the $I S O(3)$ ChernSimons gravity and Ponzano-Regge model. The $q$-deformed Ponzano-Regge model proposed by Turaev and Viro[3] is expected to be related with $\operatorname{SO}(4)$ or $\operatorname{SO}(3,1)$ Chern-Simons gauge theory and lead to Einstein gravity with a cosmological term [1] [5]. It is thus natural to extend the present formulation into the lattice gauge gravity with cosmological term and try to find the connection with the $q$-deformed version of Ponzano-Regge model. There are already several trials on these directions 17 18 but our lattice formulation may give new insights.

Since our lattice gauge gravity formulation of the present paper has natural correspondence with the Regge calculus [9], it is expected that the extension to four dimension is straightforward and is expected to be related with the $B F$ gravity theory which has 15- $j$ interpretation[19]. As in the present formulation the area of the triangle will be discretized in four dimensional lattice gauge gravity theory 20]. 
It will be interesting to extend the current formulation into the lattice version of generalized Chern-Simons gravity. The three dimensional generalized Chern-Simons action includes not only one form gauge field but also zero, two and three form gauge fields [11]. It will be interesting to ask what the role of other form gauge fields is. It should also be noted that the four dimensional generalized Chern-Simons action includes $B F$ term together with several other terms which include zero, one, two, three, and four forms.

\section{Acknowledgements}

We thank M.Fukuma, T.Kugo, L.Smolin and H.Suzuki for useful discussions and comments. This work is supported in part by Japan Society for the Promotion of Science under the grant number 09640330.

\section{References}

[1] E.Witten, Nucl.Phys.B311 (1988/89) 46.

[2] G.Ponzano and T.Regge, in Spectroscopic and group theoretical methods in physics, ed.F.Block (North-Holland, Amesterdam, 1968).

[3] V.G.Turaev and O.Y.Viro, Topology 31 (1992) 865.

[4] V.G.Turaev, C.R.Acad.Sci.Paris 313(1991) 395; J.Diff.Geom. 36 (1992) 35.

[5] H.Ooguri and N.Sasakura, Mod.Phys.Lett.A6 (1991) 3591;

H.Ooguri, Nucl.Phys.B382 (1992) 276;

N.Sasakura, Prog.Theor.Phys.Suppl.110 (1992) 191.

[6] D.Boulatov, Mod.Phys.Lett.A7 (1992) 1629.

[7] S.Chung, M.Fukuma, and A.Shapere, Int.Jour.Mod.Phys.A9 (1994) 1305.

[8] R.Anishetty, S.Cheluvaraja, H.S.Sharatchandra,M.Mathur, Phys.Lett.B314 (1993) 387.

[9] N.Kawamoto, H.B.Nielsen, Phys.Rev.D43 (1991) 1150. 
[10] M.Caselle, A.D’adda, and L.Magnea, Phys.Lett.B232 (1989) 457.

[11] N.Kawamoto and Y.Watabiki, Commun.Math.Phys. 144 (1992) 641; Mod.Phys.Lett. A7 (1992) 1137.

[12] N.Kawamoto and Y.Watabiki, Phys.Rev.D45 (1992) 605; Nucl.Phys. B396 (1993) 326.

[13] G.T.Horowitz, Commun.Math.Phys. 125 (1989) 417;

A.Ashtekar and J.D.Romano, Phys.Lett.B229 (1989) 56.

[14] T.Regge, Nuovo Cimento 19 (1961) 558.

[15] M.Creutz, Quarks, Gluons and Lattice, Cambridge University Press, 1983.

[16] D.A.Varshalovich, A.N.Moskalev, and V.K.Khersonskii, Quantum Theory of Angular Momentum (World Scientific, 1988).

[17] F.Archer and R.M.Williams, Phys.Lett. B273 (1991) 438;

S.Mizoguchi and T.Tada, Phys.Rev.Lett. 68 (1992) 1795;

B.Durhuus, H.P.Jakobsen and R.Nest, Nucl.Phys. B25 A (Proc. Suppl.) (1992) 109 ;

F.J.Archer Phys.Lett. B295 (1992) 199.

[18] D.Boulatov, Int.J.Mod.Phys. A8 (1993) 3139; Mod.Phys.Lett. A8 (1993) 3491.

[19] H.Ooguri, Mod.Phys.Lett. A7 (1992) 2799;

L.Crane and D.N.Yetter, in Quantum Topology, ed. by R.Baadhio, L.H.Kauffman, (World Scientific 1993) p120;

M.Reisenberger, Class.Quantum Grav. 14 (1997) 1753.

[20] C.Rovelli and L.Smolin, Nucl.Phys. B442 (1995) 593. 